\title{
Kritische Bemerkungen und neue Gesichtspunkte zur jungpaläolithischen Bestattung von Combe Capelle Périgord
}

\author{
Von Gisela Asmus, Köln \\ Mit 1 Abbildung im Text
}

\begin{abstract}
Zusammenfassung: Das Skelett von Combe Capelle wurde bisher in das „Untere Aurignacien" (Périgordien I der neuen Nomenklatur) datiert. Diese Datierung erscheint jedoch zweifelhaft. Die alten Grabungsmethoden waren nicht exakt genug, um mit ihnen zu endgültigen stratigraphischen Schlußfolgerungen zu kommen. Die Bestattung wurde auf dem felsigen Untergrund entdeckt, leicht eingetieft in eine Strate Moustériens und „Unteren Aurignaciens“. Aber es ist nicht erwiesen, daß der Tote auch zeitlich im „Unteren Aurignacien“ bestattet wurde. Vielmehr scheint es, als handle es sich um ein Grab aus dem „Mittleren Aurignacien“ (dem „Aurignacien typique“ neuer Nomenklatur) oder sogar aus dem "Oberen Aurignacien“ (dem Gravettien neuer Nomenklatur), das nachträglich in die genannten Schichten eingesenkt worden ist. Es ist wahrscheinlich, daß der Mann von Combe Capelle nicht den ältesten Menschenfund des jüngeren Paläolithikums schlechthin darstellt und daß er selber nicht auf französischem Boden bodenständig war.

R é s u m é : Le squelette de Combe Capelle a été daté jusqu'ici en Aurignacien inférieur (Périgordien I selon la nouvelle nomenclature). Ceci semble fort douteux. Les methodes utilisées autrefois dans les fouilles n'étaient pas assez précises. Dans ces conditions il était difficile d'aboutir à des conclusions définitives. La sépulture était trouvée sur le roc même dans une couche mince du Moustérien et de "l'Aurignacien inférieur". Mais ceci ne convainc pas, que le mort était enterré en époque de "l'Aurignacien inférieur". Il semble plûtot, qu'il s'agisse d'une tombe de "l'Aurignacien moyen" (Aurignacien typique selon la nouvelle nomenclature) ou peut être de "l'Aurignacien supérieure" (Gravettien selon la nouvelle nomenclature), qui etait enterrée ultérieurement dans les couches nommées. Il est vraisemblable, que l'homme de Combe Capelle ne représente pas l'homme le plus ancien du Paléolithique supérieur, et qu'il n'est pas originaire du sol français.
\end{abstract}

Vor 55 Jahren barg HAUSER aus Schichten des Ubergangsbereiches Moustérien — „Unteraurignacien" einen sensationellen Fund: die Bestattung von Combe Capelle. Im einzelnen soll hier auf die sattsam bekannten Fragen nach der Stellung dieses bedeutenden Fundes in der Stammesgeschichte des Menschen nicht eingegangen werden.

Nach der stratigraphischen Lagerung hielt HAUSER (in K KAATSCH u. HAUSER 1910) den Fund für „frühaurignacienzeitlich“. Er schreibt „eine Bestattung aus später paläolithischer oder gar neolithischer Epoche war von vornherein ausgeschlossen, denn im Verlauf der Ausgrabungen hatten sich die die einzelnen Niederlassungen scharf trennenden sterilen Schichten immer als völlig intakt erwiesen“ (S. 274). An anderer Stelle heißt es, es könne „keinem Zweifel unterliegen, daß der Mensch dem unteren Aurignacien angehöre“ (S. 284).

Bei genauer Durchsicht des Hausen'schen Berichtes bieten sich jedoch verschiedene Ansatzstellen, die einen so frühen Zeitpunkt der Bestattung in Frage stellen. Zunächst waren zur Zeit der Ausgrabung ganz allgemein die Grabungsmethoden noch sehr unzulänglich. Ein paläolithischer Fund erschien vom Objekt her vielfach wichtiger als die Begleitumstände seiner Lagerung. Auch muß in Betracht gezogen werden, daß vor einem halben Jahrhundert die paläolithische Kulturabfolge schematischer und einfacher erschien als heute. Manches, was heute als Parallelerscheinung aufzufassen ist, wurde damals als zeitliches Nacheinander angesehen. Inzwischen hat sich auch die Nomenklatur und die Betrachtungsweise dessen, was H.AuSER als „Aurignacien“ ansah, geändert. Jedoch kann darauf nicht eingegangen werden. Der Einfachheit halber wird hier bei der HAuser'schen Nomenklatur geblieben.

Zur Grabung selbst ließe sich folgendes bemerken: HAuser war bei der Auffindung der Bestattung von Combe Capelle nicht zugegen. Seine „durchaus zuverlässig und sorgsam ausgebildeten" Grabungsarbeiter waren bei der Auffindung des Skelettes auf sich selbst gestellt. Man darf mit Fug annehmen, daß ihnen die sachlichen Voraussetzungen für die 
richtige Beurteilung geologischer und stratigraphischer Schichtenfolgen fehlten, zumal die Verhältnisse in Combe Capelle etwas problematisch gewesen zu sein scheinen. Die dem HAUSER'schen Bericht beigegebenen Abbildungen lassen nirgends ein exaktes Grabungsprofil erkennen. HAUSER's Angaben stützen sich bei häufiger Abwesenheit großenteils auf die Aussagen seiner Grabungsarbeiter. Nach allem ergeben sich erhebliche Unsicherheitsfaktoren, die Zweifel an der bisherigen Datierung dieses wichtigen Menschenfundes als berechtigt erscheinen lassen.

Hauser zeichnete am 14. Februar 1909 ohne Angabe der Lage von dem Abri-Gelände ein seiner Veröffentlichung leider nicht beigegebenes Profil. In seinem Bericht findet sich lediglich eine Faustskizze BÄCHLER's, die dieser 7 Monate später am 12. September anfertigte. Das Hauser'sche Profil und die Bächlen'sche Skizze sollen sich in ihren Aussagen decken. Es entsteht jedoch der Eindruck, als sei die Bestattung nachträglich in die Profilskizze B̈̈CHLER's hineinprojiziert und in ihrer Lagerung interpretiert worden.

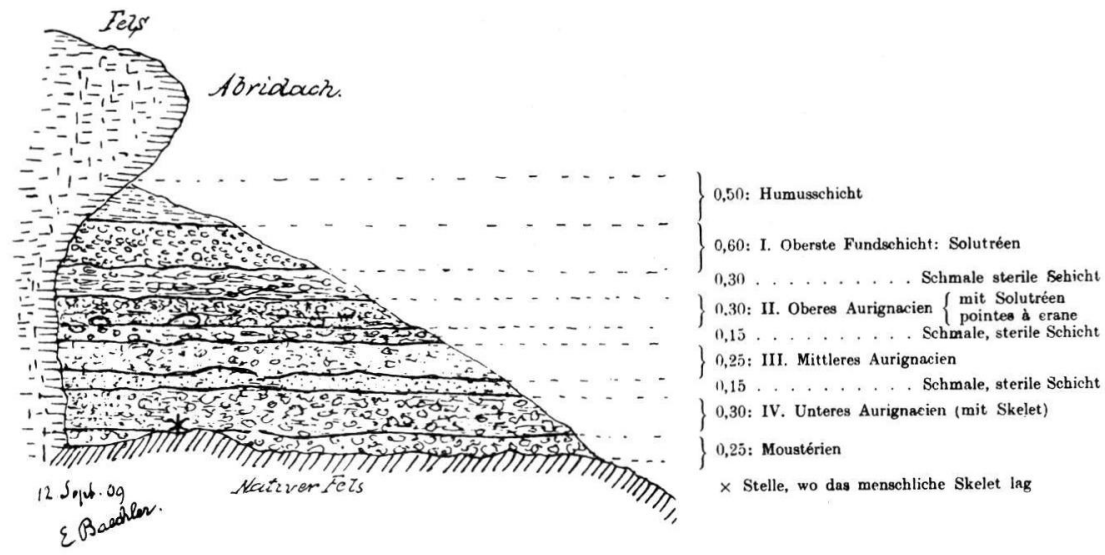

Lageskizze des Skelettes von Combe Capelle (n. KlaAtsch u. Hauser).

Hauser (1910, S. 274) schreibt über die Stratigraphie von Combe Capelle „die einzelnen Horizonte wechselten in ihrer Mächtigkeit von Woche zu Woche, und im vertikalen Profil verlor sich bald die eine oder die andere der 4 Schichten auf kurze Distanzen von $30-50 \mathrm{~cm}$. Ein für jede Schicht durchgreifendes Horizontalprofil konnte wegen der überlagernden mächtigen Felsblöcke nicht aufgeführt werden. Kontinuierlich meßbar blieben immer die Schichten I (Solutréen) und IV (unteres Aurignacien“ ... „Die Schicht II und III (oberes und mittleres Aurignacien, Verf.) ... verschwanden in lokaler Begrenzung auch wieder vom 17.-23. August 1909, um aber schon am 24. August wieder deutlich meßbar zu Tage zu treten“. Am 26. August wurde die Bestattung entdeckt und HAUSER herbeigerufen. Über das „Verschwinden“ der Schichten des „mittleren“ und „oberen" Aurignaciens äußert sich der Ausgräber nicht weiter. Er gibt auch nicht an, in welcher Lagebezeichnung zur Bestattung das Verschwinden und Wiederauftreten der Schichten II und III steht. Könnte nicht das „Verschwinden“ der Schichten mit einer bereits im Paläolithikum erfolgten Störung in Verbindung stehen? Die vermeintliche Úbereinstimmung der Profile B̈̈CHLER's und Hauser's schließt bei der dazwischenliegenden Zeitspanne und Lückenhaftigkeit der HAUSER'schen Beobachtungen und Berichte keineswegs die Annahme einer alten Störung aus, die mit der Bestattung in Zusammenhang stand. Auch besagt die Integrität der sterilen Schicht beider Profile nicht, daß die Schicht zwischen den beiden Profilen über der Bestattung selbst vorhanden und intakt war. Die B̈̈CHLER'sche Skizze läßt die Kulturschichten waagerecht verlaufen, HAUSER hingegen spricht von einer sanften Neigung der Schichten vom Kopfende der Bestattung bis zu den 
Füßen. Abgesehen davon, daß aus dieser Divergenz auf verschiedene Lokalisationen der Profile zu schließen ist, ergibt sich aus der Hanglage der Homo-Schicht ein bedeutsames Moment. Ein geneigter Schichtverband ist weniger stabil als ein waagerecht gelagerter. Durch Rutschbewegung können Verschiebungen der hangenden Schichten entstehen, die u. U .einem „Verschwinden“ einer Strate gleichkommen. Es dürfte in solchen Fällen außerordentlich schwierig sein, die unterbrochenen Schichten vor allem dann, wenn es sich um nur dünne Straten handelt, zu identifizieren.

Bei Hauser (1910, S. 284) heißt es, „es ist klar, daß die Moustérienschicht am Grunde der Grotte auf eine Strecke weit hat entfernt werden müssen, als die Aurignac-Menschen ihren Toten bestatteten. Hier mußten sich die schönsten coups de poing finden, die sie vielleicht einfach als Schmuck zu dem Toten legten." Hauser nimmt mit Recht an, daß die Bestattung in den Boden eingetieft wurde, wobei ein Teil der Grabgrube bis auf den gewachsenen Felsen ausgehoben worden ist. Seiner Annahme, daß die Homo-Schicht nach der Grablegung des Toten nicht mehr gerührt worden sei, ist beizustimmen. Aber besagt denn die Integrität der Bestattung etwas über deren Zugehörigkeit zum „unteren“ Aurignacien? Auch bei einer Bestattung aus späterer Zeit könnte ein solcher Basisbefund gegeben sein. Fest steht wohl nur, daß die Bestattung in Moustérien-Périgordien I-Schichten gefunden wurde. Der Umstand, daß die Bestattung im Abtropfbereich des Felsens lag und daß die Bergung teilweise mit einer Spitzhacke vor sich ging, läßt angesichts der geringen Schichtenstärke die Datierung „frühes Aurignacien“ als recht vage erscheinen. Es kann sich damit nur um eine Interpretation HausER's handeln, nicht aber um einen Beweis für die Richtigkeit seiner Annahme, die Bestattung stamme aus dem „unteren“ Aurignacien bzw. dem „Périgodien I“ heutiger Nomenklatur.

Am Grund der Grabgrube stellte HAUSER einen sehr auffallenden Befund fest. „Nach der Hebung des ganzen Skelettes zeigten sich im anstehenden Felsboden unverkennbare Spuren einer künstlichen Veränderung, die im Zusammenhang stand mit der Lagerung. Der vom Kopfende an sich sanft nach Süden absenkende Boden wies genau unter der Kreuzbeinregion eine Vertiefung von $6 \mathrm{~cm}$ auf, welche sich in einer Länge von $40 \mathrm{~cm}$ feststellen ließ; die Ränder dieser Vertiefung gingen einander immer parallel und das Maximum der Breite betrug $8-10 \mathrm{~cm}$. Im Grunde dieser Grube lagen die Dornfortsätze der Kreuzbeinwirbel so fest eingebettet, daß sie nach der Hebung des Beckens und des Kreuz-

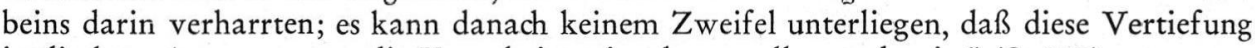
in direkter Anpassung an die Kreuzbeinregion hergestellt worden ist" (S. 277).

GIESELER (1952 und 1953) hat sich bereits mit dem merkwürdigen Grabbefund von Combe Capelle auseinandergesetzt. Er kommt zu dem Ergebnis, daß es sich um eine eindeutige Bestattung handele, aber um eine Bestattung eigener Art. Er legt dar, daß höchstwahrscheinlich ein Mann durch einen Schlag gegen die linke Schläfe getötet und bestattet wurde, nachdem er in 4-5 Teile zergliedert worden war. Der Kopf mit dem Hals und den obersten 3 Brustwirbeln ruhte in nicht gestörtem anatomischen Verband, aber von der übrigen Wirbelsäule getrennt, in völlig unnatürlicher Haltung. Die Brustregion einerseits und die Lendenregion andererseits scheinen sich bei der Grablegung ebenfalls noch in anatomischem Verband befunden zu haben. Nach den Darlegungen GIESELER's und den Abbildungen bei HAUSER sind auch die unteren Exremitäten jede für sich in die Grube gelegt worden, fanden sich doch die Oberschenkelköpfe relativ weit von den entsprechenden Gelenkpfannen entfernt. Die Lage der Fußknochen läßt darauf schließen, daß - wie bereits KLAatsch (1910) es feststellte - eine Fesselung im Bereich der Fußgelenke erfolgte. Die Füße selbst zeigen nach GIEsELER einen deutlichen Versuch, durch Einstiche von der inneren Seite des Fußes her den natürlichen Verband zu zerstören.

Im Zusammenhang mit den Beobachtungen GIESELER's erscheint der auffallende Grabungsbefund auf dem felsigen Untergrund der Grube mit ihrer künstlichen Eintiefung, in der die Dornfortsätze des Kreuzbeins hafteten, ein besonderes Gesicht zu bekommen. 
Sollte hier der Versuch unternommen worden sein, die Rückenwirbel des Toten auf irgendeine Weise am Grunde der Grabgrube zu fixieren?

Diese Beobachtungen sprechen für magische Maßnahmen, etwa die, einen Toten am Wiedergehen zu hindern. Auch ist mit Gieseler an Kannibalismus zu denken, der ebenfalls magischen Charakters gewesen sein dürfte.

Der Tote wurde anscheinend, leicht auf die rechte Seite geneigt, bestattet. Doch müssen weitere Einzelheiten offenbleiben. Die oft reproduzierte Abbildung 2 bei Hauser, die die Bestattung „in ursprünglicher Lage“ zeigt, scheint nicht den wirklichen Gegebenheiten in situ entsprochen zu haben. Tafel XXVI bei HAuser läßt in den wiedergegebenen Grabungsphotos 2,4 und 5 jedenfalls andere Lageeinzelheiten erkennen als auf HAusER's Abb. 2 (vgl. Lage des Kopfes, speziell des Hinterhauptes und Lage der Unterschenkel). Die Skizze BäCHLER's zeigt einen nach Süden offenen Abri und dessen Schichtung in einem Nord-Süd-Schnitt. Der Tote war mit dem Kopf nach Norden und den Füßen im Süden bestattet. Der Kopf lag unter dem überhängenden Felsdach, die Füße waren hangwärts gerichtet.

B ̈̈CHLER hat auf seiner Skizze die Lage des Toten summarisch durch ein Kreuz angedeutet, ohne Rücksicht darauf, daß ein Toter mehr Raum einnimmt als durch das Kreuz bezeichnet ist. Dazu handelt es sich um nur dünne Kulturschichten. Der Tote lag, leicht eingetieft in eine nur $25 \mathrm{~cm}$ starke Moustérien-Schicht, vor allem in einer Schicht "unteren“ Aurignaciens (nach BÄCHLER $30 \mathrm{~cm}$ stark). Bei Annahme einer Körpertiefe des Toten von $30-40 \mathrm{~cm}$ dürfte der Bestattete die obere Abschlußkante des Unteraurignacien-Horizontes annähernd erreicht haben. Die Stärke der hangenden sterilen Schicht gibt Bächler mit nur $15 \mathrm{~cm}$ an. Mit einer so geringen Deckschicht wäre jedoch ein Bestatteter nicht genügend gegen Raubzeug aller Art geschützt gewesen. Tatsächlich ist aber der Tote nicht in seiner Lagerung gestört worden. Daher ist wohl mit einer im ganzen stärkeren Deckschicht zu rechnen.

Die hangende Schicht des „mittleren“ Aurignaciens (nach B̈̈CHLER $25 \mathrm{~cm}$ stark) wird ebenfalls von einer nur $15 \mathrm{~cm}$ starken Schicht abgeschlossen. Wenn man der BächLER'schen Skizze vertrauen will, ist die nächstfolgende Schicht des „oberen " Aurignaciens (30 cm stark) mit größeren Steinblöcken durchsetzt. HAUSER spricht von „überlagernden mächtigen Felsblöcken", deretwegen er kein Profil von dem unmittelbaren Lageplatz der Bestattung hätte zeichnen können. Doch führt er nicht an, in welcher Schicht sich die Blöcke befanden. Eine solche Angabe dürfte bei der geringen Schichtenstärke auch schwierig gewesen sein.

Die vermeintlichen Gesteinsblöcke liegen nach B̈̈CHLER's Skizze nur etwa $85-90 \mathrm{~cm}$ über dem Moustérien-Horizont mit der Bestattung. Unter Höhenanrechnung des liegenden Toten bliebe für die Erdbedeckung bis zur Basis der Blöcke führenden Schicht noch eine Dicke von etwa $50 \mathrm{~cm}$. Eine solche Schicht, auf der noch zusätzlich Steine lagen, wäre geeignet gewesen, einen 'Toten vor Raubzeug zu schützen. Jedoch bedeutet eine derart gesicherte Grabanlage stratigraphisch, daß die Bestattung von Combe Capelle nicht im „unteren“", sondern im "mittleren“, wenn nicht gar „oberen" Aurignacien anzusetzen wäre.

Die angestellten Überlegungen führen zu dem Schluß, daß die Bestattung von Combe Capelle geologisch jünger sein dürfte, als bisher angenommen wurde. Die Lagerung in einer Grenzschicht Moustérien-Unteraurignacien an sich besagt wenig über die Zeitstellung der Bestattung. Sie sagt nur, daß versucht wurde, eine Grabanlage bis auf den gewachsenen Felsen einzutiefen. Will man B̈̈Ghler und Hauser in der Annahme folgen, die sterile Schicht über dem „oberen Aurignacien“ wäre durchgehend intakt gewesen, so würde das nur bedeuten, daß die Bestattung vor dem Solutréen vorgenommen worden ist.

Für die Frage des jungpaläolithischen Menschen ist es nicht ohne Bedeutung, ob der Mensch von Combe Capelle an den Anfang des Jungpaläolithikums zu stellen ist und 
damit den ältesten Fund eines jungpaläolithischen Menschen schlechthin darstellt (Périgordien I), oder ob er um etliche Jahrtausende jünger ist.

Merkwürdigerweise hat der Fund von Combe Capelle in Frankreich typologisch kaum Parallelen. Hingegen haben JeLineK $(1954,1955,1958,1959)$ und VLčEK $(1956,1957$, 1961) für die Funde des böhmisch-mährischen Raumes auf Combe-Capelle-Entsprechungen verwiesen. Schon KLAatsch (1910) hielt den Combe-Capelle-Fund für eine östliche Form. Der Combe-Capelle-Fund einerseits und die böhmisch-mährischen Funde andererseits gleichen einander in beträchtlichem Maße und dürften in enger Beziehung zueinander stehen. Außerdem lassen sich der Mann von Combe-Capelle und die französischen Cromagnon-Typen schwerlich in engeren typologischen Zusammenhang bringen. Nach Asmus (1951 und 1952) scheinen beide eigengeprägte Typen mit spezifischen Merkmalskombinationen zu sein und wenig morphologische Beziehungen zu haben. Die französischen Funde vertreten eine progressive Gruppe, die des böhmisch-mährischen Raumes in der Art von Combe Capelle hingegen eine viel urtümlichere. Auch mit der Annahme von Konstitutionstypen, wie GrIMM (1952/53) es in Betracht zieht, wird man dem Sachverhalt wohl nicht gerecht.

Landschaftliche Isolation führt zu Herausbildung regionaler Typengruppen. Das dürfte im Paläolithikum um so mehr der Fall gewesen sein, als die bewohnbaren Räume Europas nur dünn besiedelt waren. Auch die Annahme von Horden oder Großfamilien und die damit verbundene Endogamie unterstützen die Herausbildung regionaler Typen.

Um die Stratigraphie einiger tschechoslowakischer Menschenfunde ist es unter den Paläolithforschern der ČSR zu Meinungsverschiedenheiten gekommen. Einige Funde sollen dem Würm III angehören, andere dagegen dem Würm II. Für unsere Frage ist es weniger von Bedeutung, in welche Strate die einzelnen tschechischen Funde zu datieren sind. Bedeutungsvoller erscheint es hier, daß sogar schon im Würm II Combe-Capelle-verwandte Typen im östlichen Mitteleuropa gelebt haben könnten. Im Würm III ist dieser CombeCapelle-Typ auf alle Fälle dort anzutreffen. Anscheinend haben wir in Böhmen und Mähren ein Dichtezentrum dieses Typs. Bei seiner Vereinzelung in Frankreich und seinen häufigen Entsprechungen im östlichen Mitteleuropa liegt es nahe, den Combe-CapelleFund in engere Beziehungen zu dem letztgenannten Gebiet zu bringen und ihn als einen nach Westeuropa versprengten Ausläufer Ostmitteleuropas anzusehen.

Die jungpaläolithische Bevölkerung Alteuropas ist ungeachtet der großen Zeitabstände, verschiedenen Räume und Segregationen gelegentlich durch v. BonIN (1935) als eine biologische Einheit aufgefaßt worden. Sicherlich handelt es sich aus der Perspektive der Evolution des Menschen um "Varianten“. Diese Varianten waren aber jahrtausendelang landschaftlich isolierte und dadurch geprägte eigene Gruppen. Die jungpaläolithischen Menschen Europas als Vertreter des frühen Homo sapiens sind nicht mit der gleichen Linsenstärke anzusehen wie die Funde des Mittel- oder Altpaläolithikums.

Ebensowenig wie die Maßstäbe des Alt- und Mittelpaläolithikums können die Betrachtungsweisen moderner Populationen auf den jungpaläolithischen Menschen angewandt werden. Schlüsse, die dahinzielen, die jungpaläolithischen Menschenfunde Europas variierten statistisch-mathematisch nicht mehr als die Bevölkerung des heutigen Europa und entsprächen damit im wesentlichen heutigen Populationen, gehen (von der Zweifelhaftigkeit des Wertes von Variationskoeffizienten ganz abgesehen) an wesentlichen Punkten vorüber. Das dünnbesiedelte Europa des Jungpaläolithikums bot infolge seiner durch Eisbarrieren eingeengten und abgeschlossenen Räume ganz andere Isolationsmöglichkeiten als je wieder in späterer Zeit. Für die Herausbildung spezifischer Gruppen des frühen Homo sapiens ergaben sich geradezu ideale Voraussetzungen.

Wanderungen im heutigen Sinn werden für das Paläolithikum nicht anzunehmen sein. Der jungpaläolithische Mensch war seinem Status nach schweifender Jäger und als solcher weitgehend von den Gewohnheiten und jahreszeitlich bedingten Zügen "seines" Wildes 
abhängig. Die stellenweise gefundenen Reste von Hüttenbauten oder Zelten lassen wohl nur ein vorübergehendes Verweilen an einem Ort erschließen. Allgemein wird mit einem Fluktuieren von Großfamilien und mit rhythmischen Zügen durch große Räume zu rechnen sein, die sich auf landschaftsbedingten natürlichen Wegen vollzogen. Im Laufe der Jahrtausende werden sich, ausgehend von ursprünglichen Wildwechseln, Wanderwege herausgebildet haben, auf denen im Laufe der Zeit west-östliche Wechselbeziehungen zustande gekommen sein mögen.

Mit dem Offinen der ursprünglich eisbedingten Isolationsräume boten sich zwanglos Möglichkeiten der Kontaktnahme und Durchdringung östlicher und westlicher Kulturen. Bei einer schweifenden Jägerbevölkerung wird es relativ kurzer Zeit bedurft haben, um von dem kontinental beeinflußten östlichen Mitteleuropa in das klimatisch günstigere atlantisch beeinflußte Westeuropa zu gelangen. Sollte der Mann von Combe Capelle direkt oder indirekt mitteleuropäischen Ursprungs gewesen sein, so wird ihn wahrscheinlich nicht eine gezielte Wanderung nach Frankreich geführt haben. Doch liegt es durchaus im Bereich des Möglichen, daß er im Zuge des günstigeren Temperaturgefälles nach Südwesten dorthin gelangte.

\section{Schriftenverzeichnis}

Asmus, G.: Neandertaler und Homo sapiens. - Eiszeitalter und Gegenwart 1, Óhringen 1951. - Zur Frage der jungpaläolithischen Brünn-Rasse. - Homo 3, Göttingen 1952.

v. Bonin, G.: European races of upper palaeolithic. - Human Biology 7, Baltimore 1935.

GIEsELER, W.: Schädelverletzungen, Kannibalismus und Bestattungen im europäischen Paläolithikum. - Aus der Heimat 60, Öhringen 1952. - -Das jungpaläolithische Skelett von Neuessing. - Aus der Heimat 61, Öhringen 1953.

Grimm, H.: Altes und Neues von Aurignac-Typus. - Wiss. Z. Univ. Halle-Wittenberg 2. Math.nat. Reihe 6, 1952/53

Jelinek, J. The fossil man of Dolni Vestonice III (Czechoslovakia). - Anthropozoikum 3, Prag 1954. - - Der jungpaläolithische Skelettfund von Unter-Wisternitz. - Mitt. Anthrop. Ges. Wien. 84/85, Wien 1955 - - Neue Funde und Datierungen des paläolithischen Menschen in der Tschechoslowakei. - HOMO, 9, Berlin/Frankfurt 1958. - - Der fossile Mensch Brno II. - Anthropos, N.S. 1, Brno 1959.

KLAatsch, H. und O. HausER: Homo aurignacensis Hauseri. Ein paläolithischer Skelettfund aus dem unteren Aurignacien der Station Combe Capelle bei Montferrant (Périgord). Prähist. Z. 1, Berlin 1910.

Kuıma, B.: Die Entdeckung menschlicher Skelettreste aus dem oberen Paläolithikum in Pavlov, Südmähren. - Mitt. Anthrop. Ges. Wien, 88/89, Wien 1959.

VLčEK, E.: Kalva pleistocénniho člověka z Podbaby (Praha XIX). - Anthropozoikum 5, Prag 1956. - - Pleistocénni člověk z jeskyně na Zlatém Koni u Koněprus. - Anthropozoikum 6, Prag 1957. - - Pozustatky mladopleistocénniho člověka z Pavlova. - Památky archeologické 52, 1. (Festschrift für Jaroslav Böhm), Prag 1961.

Manuskr. eingeg. 21. 2. 1964.

Anschrift der Verf.: Dr. Gisela Asmus, 5. Köln-Lindenthal, Weyertal 125. 